\title{
Beneficial Effects of an 8-Week, Very Low Carbohydrate Diet Intervention on Obese Subjects
}

\author{
Yunjuan Gu, ${ }^{1,2,3,4,5}$ Haoyong Yu, ${ }^{1,2,3,4}$ Yuehua Li, ${ }^{4}$ Xiaojing Ma, ${ }^{1,2,3,4}$ Junxi Lu, ${ }^{1,2,3,4}$ \\ Weihui Yu, ${ }^{1,2,3,4}$ Yunfeng Xiao, ${ }^{4}$ Yuqian Bao, ${ }^{1,2,3,4}$ and Weiping Jia ${ }^{1,2,3,4}$ \\ ${ }^{1}$ Department of Endocrinology and Metabolism, Shanghai Jiao Tong University Affiliated Sixth People's Hospital, \\ Shanghai Diabetes Institute, Shanghai Clinical Centre of Diabetes, Shanghai 200233, China \\ ${ }^{2}$ Shanghai Key Laboratory of Diabetes Mellitus, Shanghai 200233, China \\ ${ }^{3}$ Department of Endocrinology and Metabolism, Affiliated Hospital of Nantong University, Nantong 226001, Jiangsu Province, China \\ ${ }^{4}$ Department of Radiology, Shanghai Jiao Tong University Affiliated Sixth People's Hospital, Shanghai 200233, China \\ ${ }^{5}$ Department of Endocrinology and Metabolism, Affiliated Hospital of Nantong University, Nantong, Jiangsu Province, China
}

Correspondence should be addressed to Weiping Jia; wpjia@sjtu.edu.cn

Received 31 December 2012; Revised 18 February 2013; Accepted 18 February 2013

Academic Editor: Wei Jia

Copyright (C) 2013 Yunjuan Gu et al. This is an open access article distributed under the Creative Commons Attribution License, which permits unrestricted use, distribution, and reproduction in any medium, provided the original work is properly cited.

Aim. To investigate the effects of weight loss during an 8-week very low carbohydrate diet (VLCD) on improvement of metabolic parameters, adipose distribution and body composition, and insulin resistance and sensitivity in Chinese obese subjects. Methods. Fifty-three healthy obese volunteers were given an 8-week VLCD. The outcomes were changes in anthropometry, body composition, metabolic profile, abdominal fat distribution, liver fat percent (LFP), and insulin resistance and sensitivity. Results. A total of 46 (86.8\%) obese subjects completed the study. The VLCD caused a weight loss of $-8.7 \pm 0.6 \mathrm{~kg}$ (mean \pm standard error (SE), $P<$ 0.0001 ) combined with a significant improvement of metabolic profile. In both male and female, nonesterified fatty acid (NEFA) significantly decreased $(-166.2 \pm 47.6 \mu \mathrm{mol} / \mathrm{L}, P=0.001)$ and $\beta$-hydroxybutyric acid $(\mathrm{BHA})$ increased $(0.15 \pm 0.06 \mathrm{mmol} / \mathrm{L}, P=$ 0.004) after eight weeks of VLCD intervention. The significant reductions in subcutaneous fat area (SFA), visceral fat area (VFA), and LFP were $-66.5 \pm 7.9 \mathrm{~cm}^{2},-35.3 \pm 3.9 \mathrm{~cm}^{2}$, and $-16.4 \pm 2.4 \%$, respectively (all $P$ values $P<0.0001$ ). HOMA IR and HOMA $\beta$ significantly decreased while whole body insulin sensitivity index (WBISI) increased (all $P$ values $P<0.001$ ). Conclusion. Eight weeks of VLCD was an effective intervention in obese subjects. These beneficial effects may be associated with enhanced hepatic and whole-body lipolysis and oxidation.

\section{Introduction}

In the past decades, the prevalence of overweight and obesity has increased rapidly worldwide. Obesity is a major risk factor for several chronic diseases such as type 2 diabetes mellitus, cardiovascular disease, and some kinds of cancer [1]. Because of the severe comorbidities of obesity, people attempt to lose weight through several alternative diets. Recently, there has been a resurgence of interest in VLCD as a means of weight loss and metabolic improvements. Evidence from clinical studies and meta-analyses suggested that VLCD can decrease body weight, improve metabolic parameters, insulin resistance/sensitivity, and nonalcoholic fatty liver disease (NAFLD) [2]. However, the effect of VLCD on $\beta$ cell function and insulin sensitivity is still controversial. Svendsen et al. reported that when ten obese women lost $11 \%$ of their baseline weight after an 8-week VLCD diet, their subcutaneous and intra-abdominal fatty tissue significantly decreased, but HOMA IR increased and peripheral insulin sensitivity was unaltered [3]. Malandrucco et al. observed that the metabolic profile of 14 severely obese patients with type 2 diabetes with a 7-day VLCD intervention were improved markedly primarily due to the amelioration of $\beta$ cell function (disposition index increased), but with no change in insulin sensitivity [4]. Viljanen et al. found that after 16 obese subjects underwent a 6-week VLCD, visceral adipose and abdominal subcutaneous fat decreased while whole body insulin sensitivity increased, but adipose tissue regional glucose uptake 
remained unchanged [5]. Therefore, it is necessary to further assess the beneficial effect of VLCD, especially its impact on $\beta$ cell function and insulin sensitivity.

It has been reported that the amount of carbohydrate intake affects blood glucose and insulin levels and even metabolic consequences [6]. However, in traditional Chinese dietary, carbohydrate contributes more than half of the total daily energy consumption. The method of VLCD to lose weight is less popular in Chinese comparing with that in westerns. Therefore, this is the first time to investigate the beneficial effects of VLCD on weight loss, improvements of body composition, insulin sensitivity and resistant, and plasma metabolic parameters in Chinese obese subjects.

\section{Methods}

2.1. Participants. We recruited 53 obese healthy volunteers (31 men, 22 women, aged $18-52$ years, BMI $\geq 28 \mathrm{~kg} / \mathrm{m}^{2}$ ) from the outpatient clinic of endocrinology and metabolism department of Shanghai Jiao Tong University Affiliated Sixth People's Hospital. All the subjects underwent a general health examination before entering the study. Exclusion criteria were as follows: pregnant or plan for pregnant; lactation or postmenopausal women; use of any prescription medication in previous 2 months; had any weight loss diet or pill during the past 6 months; consuming $>20$ g/day of alcohol; tobacco use within 6 month; cardiovascular or endocrine disease history; hypertension history or current elevated blood pressure (systolic blood pressure (SBP): $\geq 150 \mathrm{~mm} \mathrm{Hg}$; diastolic blood pressure (DBP) $\geq 90 \mathrm{~mm} \mathrm{Hg}$; current treatment for hypertension); diabetes mellitus; acute or chronic infections; hepatopathy, kidney disease, gastrointestinal disease or any other acute or chronic diseases requiring treatment.

This study was approved by the institutional review board of Shanghai Jiao Tong University Affiliated Sixth People's Hospital in accordance with the principles of the Helsinki Declaration II. Written informed consent was obtained from each participant. There were no monetary incentives for participation.

2.2. Experimental Protocol. One week before initiation of the study, all subjects were asked to maintain their habitual energy intake. At baseline and after the 8-week VLCD treatment, standard 75-g oral glucose tolerance tests (OGTT) were performed. At weeks 0, 4, and 8, anthropometric parameters, bioimpedance assessments, biochemical indices, insulin resistance and sensitivity, LFP, SFA, and VFA were measured.

2.3. Dietary Intervention. All subjects were given individual instructions on how to follow the VLCD. Energy intake was restricted to less than $800 \mathrm{kcal} /$ day (carbohydrate intake < $20 \mathrm{~g} / \mathrm{d}$ ). All daily meals were replaced as follows: a cup of soybean milk $(200 \mathrm{~mL})$ and a boiled egg at breakfast; a diet nutrition bar (106 Kcal: $2.8 \mathrm{~g}$ carbohydrate, $11.2 \mathrm{~g}$ protein and
5.6 g fat; Nutriease Health Technology Co., Ltd., Hangzhou, China), nonstarchy vegetables $(<200 \mathrm{kcal})$, and $50 \mathrm{~g}$ protein from meat (i.e., beef, lean pork, skinned chicken, fish) at lunch and dinner. Supplementation of multivitamins and minerals was provided per day. Subjects were also encouraged to drink at least 1.8 litres of water per day, and asked to maintain their habitual level of physical activity. Regular telephone contact to individual by nutritionists was provided for nutrient support. After the 8-week dietary intervention, there was a 1-week recovery period when the eucaloric intake was allowed to overcome the catabolic state. Then participants restarted normal eating and were provided with information about portion size and healthy eating.

\subsection{Measurements}

2.4.1. Bioimpedance Assessments. Before and after the VLCD, whole body skeletal muscle mass and fat mass were measured by a multifrequency impedance plethysmograph body composition analyzer (InBody 720, Biospace, Korea). Before analyzing, participants stand on the electrodes embedded in the scale platform of the respective analyzers and wipe the bottom of their feet with a proprietary electrolyte tissue. Then they were asked to stand upright grasping the handles of the analyzer in order to contact with 4 pairs of electrodes (octapolar technology). Resistance was measured at five specific frequencies $(1 \mathrm{kHz}, 50 \mathrm{kHz}, 250 \mathrm{kHz}, 500 \mathrm{kHz}$, and $1 \mathrm{MHz})$ and reactance at three specific frequencies $(5 \mathrm{kHz}, 50 \mathrm{kHz}$, and $250 \mathrm{kHz}$ ). Total body water (TBW) was estimated from area, volume, length, impedance, and a constant proportion (specific resistivity). Fat-free mass was estimated by dividing TBW by 0.73 . After the participant's identification number, sex, age, and height were typed into the analyzer; the fat mass percentage (FMP) and skeletal muscle mass percentage of whole body weight (SMMP) were calculated by the computer software automatically [7].

2.4.2. 1H Magnetic Resonance Spectroscopy (1H MRS) Measurement of Liver Fat Percent. Liver magnetic resonance imaging and in vivo single-voxel MRS were performed using Philips Achieva 3.0T MRI system (Philips Medical Systems, Eindhoven, The Netherlands) equipped with an 8-channel phase coil. Anatomical T1-weighted spin-echo MR images were obtained using the following parameters: repetition time $(\mathrm{TR})=550 \mathrm{~ms}$; echo time $(\mathrm{TE})=10 \mathrm{~ms}$; flip angle $=60$; field of view $(\mathrm{FOV})=21 \mathrm{~cm}$; slice thickness $=3 \mathrm{~mm}$; slice spacing $=$ $0.1 \mathrm{~mm} .{ }^{1} \mathrm{H}$-MRS was performed for quantification analysis of metabolite concentrations in liver. Firstly, 2D-spin-echo images in the coronal and sagittal regions were obtained for image-guided localization of voxels of interest (VOI) for spectroscopic data acquisition. Secondary, single-voxel MRS was performed by a stimulated echo acquisition mode (STEAM) sequence by using the following parameters: $\mathrm{TE}=$ $20 \mathrm{~ms}$; TR = 1,500 ms; VOX = $15 \times 15 \times 15 \mathrm{~mm}$; total number of points $=128$; total number of average $=64$. Finally, eightstep phase cycling was used to suppress unwanted signals or artifacts. Signal intensities of the water peak at 4.7 p.p.m. (Sw) and the fat peak at 1.2 p.p.m. (Sf) were measured and liver fat 
percentage $(\mathrm{LFP})$ was calculated as follows: $\mathrm{LFP}=100 \times \mathrm{Sf} /(\mathrm{Sf}$ $+\mathrm{Sw}$ ) [8]. A LFP of $5.56 \%$ by $1 \mathrm{H}$ MRS is used as cutoff value for diagnosing NAFLD [9].

2.4.3. Magnetic Resonance Imaging (MRI) Measurement of Abdominal Fat Areas. SFA and VFA were assessed by Philips Achieva 3.0T MRI system (Philips Medical Systems, Eindhoven, The Netherlands) using standard array coils with the subject supine. Breath-hold FISP images were centered on the L4-L5 intervertebral disc using standard localizer images with the following parameters: $\mathrm{TR}=4 \mathrm{~ms}, \mathrm{TE}=2 \mathrm{~ms}$, number of slices $=12$, slice thickness $=8 \mathrm{~mm}$, image matrix $256 \times$ 256 , and field-of-view $=500 \times 500 \mathrm{~mm}$. The 4 slices that were best aligned with the L4-L5 disc were analyzed by SliceOmatic 5.0 software package (Escape Medical Viewer V3.2) to define VFA and SFA [10]. VFA and SFA were measured by fitting a spline curve to points on the border of the subcutaneous and visceral regions. Nonfat regions within the visceral region were also outlined with a spline fit and subtracted from the total visceral region [11].

2.4.4. Laboratory Evaluation. At baseline and week 8, all participants underwent a 75-g OGTT in the morning after $10 \mathrm{~h}$ overnight fasting. Their venous blood samples were drawn at 0 (fating state), 30, 60, and 120 minutes. Plasma glucose concentration was measured using a glucose oxidase method. Serum insulin levels were tested by a radioimmunoassay using the insulin detection kit, according to the manufacturer's instructions (Beijing North Institute of Biological Technology, Beijing, China). The following indices were measured by standard commercial methods on a parallel, multichannel analyzer (Hitachi 7600-020, Tokyo, Japan): liver function panel including aspartate aminotransferase (AST), alanine aminotransferase (ALT), $\gamma$-glutamyl transferase $(\gamma$ GT); renal function panel including serum creatinine, uric creatinine, and uric acid; lipid panel including total cholesterol (TC), triacylglycerol (TG), high density lipoprotein (HDL), and low-density lipoprotein cholesterol (LDL); NEFA and BHA. Urinary albumin were tested using the firstmorning void urine samples by immunonephelometry on the BN II analyzer (Siemens Diagnostics) and its concentration was then corrected by urine creatinine.

2.5. Calculations. $\beta$ cell function and insulin resistance were evaluated using the following formulas: (1) HOMA $\beta$ $(\mathrm{mIU} / \mathrm{mmol} / \mathrm{L})=20 \times$ fasting insulin $(\mathrm{mIU} / \mathrm{L}) /($ fasting glucose $(\mathrm{mmol} / \mathrm{L})-3.5)[12]$; (2) HOMA IR $\left(\mathrm{mIU} \cdot \mathrm{mmol} / \mathrm{L}^{2}\right)=$ fasting insulin $(\mathrm{mIU} / \mathrm{L}) \times$ fasting glucose $(\mathrm{mmol} / \mathrm{L}) / 22.5[13]$; (3) Whole Body Insulin Sensitivity Index (WBISI, $\mathrm{L}^{2} / \mathrm{mmol}^{2}$ ) $=10000 /$ square root of [fasting glucose $(\mathrm{mmol} / \mathrm{L}) \times$ fasting insulin $(\mathrm{mIU} / \mathrm{L}) \times$ mean insulin $(\mathrm{mIU} / \mathrm{L}) \times$ mean glucose $(\mathrm{mmol} / \mathrm{L})]$; mean glucose $=[$ fasting glucose $(\mathrm{mmol} / \mathrm{L})+2 \mathrm{~h}$ postprandial glucose $(\mathrm{mmol} / \mathrm{L})] / 2$; mean insulin $=[$ fasting insulin $(\mathrm{mIU} / \mathrm{L})+2 \mathrm{~h}$ postprandial insulin $(\mathrm{mIU} / \mathrm{L})] / 2[14]$.

2.6. Statistical Analysis. All continuous variables were tested for normal distribution by the Kolmogorov-Smirnov normality test. Log-transformations were used to normalize skewed variables. Data were presented as mean \pm SE. Chisquare test was used for categorical variables, repeatedmeasures ANOVA for comparison among different time points, and paired $t$-test for comparison of variables before and after the study. $P$ values $<0.05$ (2-tailed tests) were considered to be statistically significant. Statistical analysis was performed using SPSS 17.0 (SPSS, Chicago, IL, USA).

\section{Results}

Of 53 obese subjects, 46 (86.8\%) completed the study. Among the 7 participants who withdrew, one was diagnosed of type 2 diabetes, one had urine microalbumin/creatinine $\geq 30 \mathrm{ug} / \mathrm{mg}$ at baseline, two dropped out for stomachache, and three for noncompliance.

3.1. Body Weight, Waist, and Blood Pressure. As shown in Table 1, after the 8-week VLCD intervention, from baseline, mean weight loss was $-8.7 \pm 0.6 \mathrm{~kg}(-9.0 \pm 0.5 \%, P<0.0001)$, representing a mean reduction in BMI of $-3.0 \pm 0.2 \mathrm{~kg} / \mathrm{m}^{2}$ $(-9.0 \pm 0.5 \%, P<0.0001)$. Mean waist circumference change was $-5.9 \pm 1.2 \mathrm{~cm}$ (from $104.5 \pm 1.7 \mathrm{~cm}$ to $98.6 \pm 1.4 \mathrm{~cm}$, $P<0.0001)$, mean reductions in SBP and DBP were $-13.1 \pm$ $2.8 \mathrm{mmHg}$ and $-8.2 \pm 1.6 \mathrm{mmHg}$ respectively (all $P$ values $<$ $0.0001)$.

3.2. Body Composition and Liver Fat Percent. As shown in Table 2, significant decrement in mean SFA $\left(-66.5 \pm 7.9 \mathrm{~cm}^{2}\right.$, $P<0.0001)$ and VFA $\left(-35.3 \pm 3.9 \mathrm{~cm}^{2}, P<0.0001\right)$ was observed after the 8 -week VLCD diet. FMP significantly decreased $(-3.0 \pm 0.4 \%, P<0.0001)$ while SMMP significantly increased $(1.7 \pm 0.2 \%, P<0.0001)$. Moreover, with LFP significantly decreasing $(-16.4 \pm 2.4 \%, P<0.0001)$, about half of the subjects who were NAFLD at baseline experienced a reversal at the end of the study (from $87.0 \%$ to $43.5 \%$, $P=0.021$ ). Furthermore, all the improvements of body composition and liver fat percent were significantly presented in both male and female subjects (Figure 1).

3.3. Plasma Metabolic Parameters and Hepatic Enzymes Concentrations. Together with weight loss from baseline to the end of the study, liver enzymes of ALT, AST, $\gamma$-GT were all significantly improved (Table 1). Concentrations of TC (from $5.12 \pm 0.12 \mathrm{mmol} / \mathrm{L}$ to $4.79 \pm 0.14 \mathrm{mmol} / \mathrm{L}, P<0.0001)$ and TG (from $1.98 \pm 0.23 \mathrm{mmol} / \mathrm{L}$ to $1.08 \pm 0.09 \mathrm{mmol} / \mathrm{L}$, $P<0.0001)$ significantly decreased and HDL significantly increased (from $1.15 \pm 0.04 \mathrm{mmol} / \mathrm{L}$ to $1.19 \pm 0.04, P=0.01$ ). However, the LDL level did not significantly change after the dietary intervention. The level of NEFA did not change at week 4 but decreased significantly at week 8 (baseline: $729.8 \pm 34.9 \mu \mathrm{mmol} / \mathrm{L}$ versus week $4: 729.3 \pm 43.2 \mu \mathrm{mmol} / \mathrm{L}$ versus week $8: 574.7 \pm 37.0 \mu \mathrm{mmol} /, P=0.001)$. Although the concentration of BHA significantly increased at week 4 (baseline: $0.06 \pm 0.01 \mathrm{mmol} / \mathrm{L}$ versus week $4: 0.56 \pm 0.13 \mathrm{mmol} / \mathrm{L}$, $P<0.0001$ ), it subsequently decreased at the end of study, with its concentration remaining significantly higher than that at baseline (baseline: $0.06 \pm 0.01 \mathrm{mmol} / \mathrm{L}$ versus week 8 : $0.24 \pm 0.07 \mathrm{mmol} / \mathrm{L}, P=0.004)$. As concerned with renal 
TABLE 1: Effects of anthropometric and biochemical parameters in obese subjects after a VLCD intervention.

\begin{tabular}{|c|c|c|c|c|c|}
\hline & Baseline & Week 4 & Week 8 & $\begin{array}{c}\text { Change from baseline to } \\
\text { Week } 8\end{array}$ & $P$ \\
\hline Weight, Kg & $96.1 \pm 2.7$ & $90.1 \pm 2.6^{*}$ & $87.4 \pm 2.5^{*}$ & $-8.7 \pm 0.6$ & $<0.0001$ \\
\hline BMI, $\mathrm{Kg} / \mathrm{m}^{2}$ & $32.6 \pm 0.6$ & $30.6 \pm 0.6^{*}$ & $29.7 \pm 0.6^{*}$ & $-3.0 \pm 0.2$ & $<0.0001$ \\
\hline Waist, cm & $104.5 \pm 1.7$ & $100.7 \pm 1.5^{*}$ & $98.6 \pm 1.4^{*}$ & $-5.9 \pm 1.2$ & $<0.0001$ \\
\hline SBP, mmHg & $135.2 \pm 2.8$ & $124.1 \pm 2.0^{*}$ & $121.7 \pm 2.0^{*}$ & $-13.1 \pm 2.8$ & $<0.0001$ \\
\hline $\mathrm{DBP}, \mathrm{mmHg}$ & $82.7 \pm 1.7$ & $76.7 \pm 1.5^{*}$ & $73.9 \pm 1.6^{*}$ & $-8.2 \pm 1.6$ & $<0.0001$ \\
\hline ALT, U/L & $42.9 \pm 5.1$ & $31.6 \pm 3.5^{*}$ & $22.3 \pm 1.6^{*}$ & $-20.6 \pm 5.2$ & 0.002 \\
\hline AST, U/L & $26.2 \pm 1.9$ & $23.0 \pm 1.5^{* *}$ & $20.3 \pm 1.5^{*}$ & $-6.0 \pm 1.7$ & 0.001 \\
\hline$\gamma-\mathrm{GT}, \mathrm{U} / \mathrm{L}$ & $55.1 \pm 6.5$ & $27.2 \pm 2.4^{*}$ & $27.8 \pm 3.5^{*}$ & $-30.4 \pm 6.3$ & $<0.0001$ \\
\hline Serum uric acid, $\mu \mathrm{mol} / \mathrm{L}$ & $395.1 \pm 13.5$ & $382.5 \pm 14.2$ & $369.2 \pm 17.0^{* *}$ & $-26.1 \pm 10.0$ & 0.035 \\
\hline $\mathrm{UACR}, \mu \mathrm{g} / \mathrm{mg}$ & $8.7 \pm 1.0$ & $7.5 \pm 0.9$ & $7.1 \pm 0.7$ & $-1.9 \pm 0.9$ & 0.059 \\
\hline $\mathrm{TC}, \mathrm{mmol} / \mathrm{L}$ & $5.12 \pm 0.12$ & $4.60 \pm 0.13^{*}$ & $4.79 \pm 0.14^{*}$ & $-0.37 \pm 0.14$ & $<0.0001$ \\
\hline $\mathrm{TG}, \mathrm{mmol} / \mathrm{L}$ & $1.98 \pm 0.23$ & $1.11 \pm 0.10^{*}$ & $1.08 \pm 0.09^{*}$ & $-0.98 \pm 0.23$ & $<0.0001$ \\
\hline $\mathrm{HDL}, \mathrm{mmol} / \mathrm{L}$ & $1.15 \pm 0.04$ & $1.09 \pm 0.03^{* *}$ & $1.19 \pm 0.04$ & $0.02 \pm 0.03$ & 0.01 \\
\hline $\mathrm{LDL}, \mathrm{mmol} / \mathrm{L}$ & $3.09 \pm 0.12$ & $3.01 \pm 0.12$ & $3.13 \pm 0.13$ & $0.05 \pm 0.10$ & 0.407 \\
\hline $\mathrm{NEFA}, \mu \mathrm{mmol} / \mathrm{L}$ & $729.8 \pm 34.9$ & $729.3 \pm 43.2$ & $574.7 \pm 37.0^{*}$ & $-166.2 \pm 47.6$ & 0.001 \\
\hline $\mathrm{BHA}, \mathrm{mmol} / \mathrm{L}$ & $0.06 \pm 0.01$ & $0.56 \pm 0.13^{*}$ & $0.24 \pm 0.07^{*}$ & $0.15 \pm 0.06$ & 0.004 \\
\hline
\end{tabular}

Data are presented as mean \pm standard error (SE). BMI: body mass index; SBP: systolic blood pressure; DBP: systolic bold pressure; TC: total cholesterol; TG: triacylglycerol; HDL: high density lipoprotein; LDL: low density lipoprotein; NEFA: non-esterified fatty acid; BHA: $\beta$-hydroxybutyric acid; ALT: alanine aminotransferase; AST: aspartate aminotransferase; $\gamma$-GT: $\gamma$-glutamyl transferase; UACR: urine microalbumin creatinine ratio. $P$ : changes of sequential data within experiments from baseline to week 8 . Significant difference observed versus baseline: ${ }^{*} P<0.001 ;{ }^{* *} P<0.05$.

TABLE 2: Effects of fat distribution, glucose homeostasis, and insulin sensitivity in obese subjects after a VLCD intervention.

\begin{tabular}{|c|c|c|c|c|c|}
\hline & Baseline & Week 4 & Week 8 & $\begin{array}{l}\text { Change from baseline to } \\
\text { week } 8\end{array}$ & $P$ \\
\hline $\mathrm{SFA}, \mathrm{cm}^{2}$ & $348.0 \pm 16.7$ & $299.4 \pm 16.2$ & $264.0 \pm 16.4$ & $-66.5 \pm 7.9$ & $<0.0001$ \\
\hline $\mathrm{VFA}, \mathrm{cm}^{2}$ & $113.9 \pm 5.8$ & $96.6 \pm 4.9$ & $79.8 \pm 3.7$ & $-35.3 \pm 3.9$ & $<0.0001$ \\
\hline Liver fat, $\%$ & $22.3 \pm 2.7$ & $13.5 \pm 3.2$ & $6.5 \pm 1.4$ & $-16.4 \pm 2.4$ & $<0.0001$ \\
\hline SMMP, \% & $33.7 \pm 0.9$ & $34.1 \pm 1.3$ & $36.7 \pm 0.8$ & $1.7 \pm 0.2$ & $<0.0001$ \\
\hline FMP, \% & $37.2 \pm 1.4$ & $38.9 \pm 2.0$ & $34.3 \pm 1.3$ & $-3.0 \pm 0.4$ & $<0.0001$ \\
\hline FPG, mmol/L & $5.3 \pm 0.1$ & $5.0 \pm 0.1$ & $5.2 \pm 0.1$ & $-0.07 \pm 0.15$ & 0.001 \\
\hline 2h PG, mmol/L & $7.5 \pm 0.3$ & - & $6.9 \pm 0.3$ & $-0.59 \pm 0.31$ & 0.061 \\
\hline FINS, $\mu \mathrm{U} / \mathrm{mL}$ & $22.5 \pm 4.0$ & $10.2 \pm 0.8$ & $11.8 \pm 1.4$ & $-11.5 \pm 4.3$ & 0.003 \\
\hline $2 \mathrm{~h}$ INS, $\mu \mathrm{U} / \mathrm{mL}$ & $134.2 \pm 11.3$ & - & $71.1 \pm 8.2$ & $-57.2 \pm 11.3$ & $<0.0001$ \\
\hline HOMA IR & $6.1 \pm 1.5$ & $2.3 \pm 0.2$ & $2.8 \pm 0.4$ & $-3.6 \pm 1.7$ & 0.006 \\
\hline HOMA $\beta$ & $235.7 \pm 21.6$ & $140.3 \pm 11.4$ & $136.2 \pm 12.1$ & $-104.8 \pm 18.3$ & $<0.0001$ \\
\hline WBISI & $67.0 \pm 6.9$ & - & $123.7 \pm 12.8$ & $54.2 \pm 13.9$ & $<0.0001$ \\
\hline
\end{tabular}

Data are presented as mean \pm standard error (SE). SFA: subcutaneous fat area; VFA: visceral fat area; SMMP: skeletal muscle mass percentage of whole body weight; FMP: fat mass percentage of whole body weight;. FPG: fasting plasma glucose; $2 \mathrm{hPG:} 2 \mathrm{~h}$ postprandial glucose; FINS: fasting insulin; WBISI: whole body insulin sensitivity index. $P$ : changes of sequential data within experiments from baseline to week 8 . Significant difference observed versus baseline: ${ }^{*} P<0.001$; ${ }^{* *} P<0.05$.

function, we observed the serum uric acid level significantly reduced (baseline: $395.1 \pm 13.5 \mu \mathrm{mol} / \mathrm{L}$ versus week $8: 369.2 \pm$ $17.0 \mu \mathrm{mol} / \mathrm{L}, P=0.035)$, but urine microalbumin creatinine ratio (UACR) did not significantly decrease (baseline: $8.7 \pm$ $1.0 \mu \mathrm{g} / \mathrm{mg}$ versus week 8: $7.1 \pm 0.7 \mu \mathrm{g} / \mathrm{mg}, P=0.059)$.

3.4. Glucose Homeostasis and Insulin Sensitivity. As shown in Table 2, significant reductions in fasting plasma glucose
(FPG), fasting insulin (FINS), and $2 \mathrm{~h}$ postprandial insulin (2hINS) were observed (all $P$ values $<0.01$ ). However, the change of $2 \mathrm{~h}$ postprandial plasma glucose (2hPG) was not statisticaly significant at the end of the study $(P=0.061)$. HOMA IR and HOMA $\beta$ significantly improved with the reduction of $-3.6 \pm 1.7$ and $-104.8 \pm 18.3$ (all $P$ values $<$ $0.0001)$, respectively. WBISI significantly increased from $67.0 \pm 6.9$ to $123.7 \pm 12.8(P<0.0001)$. Moreover, as shown in Figure 2, FINS, 2hINS, HOMA IR, HOMA $\beta$, and WBISI 

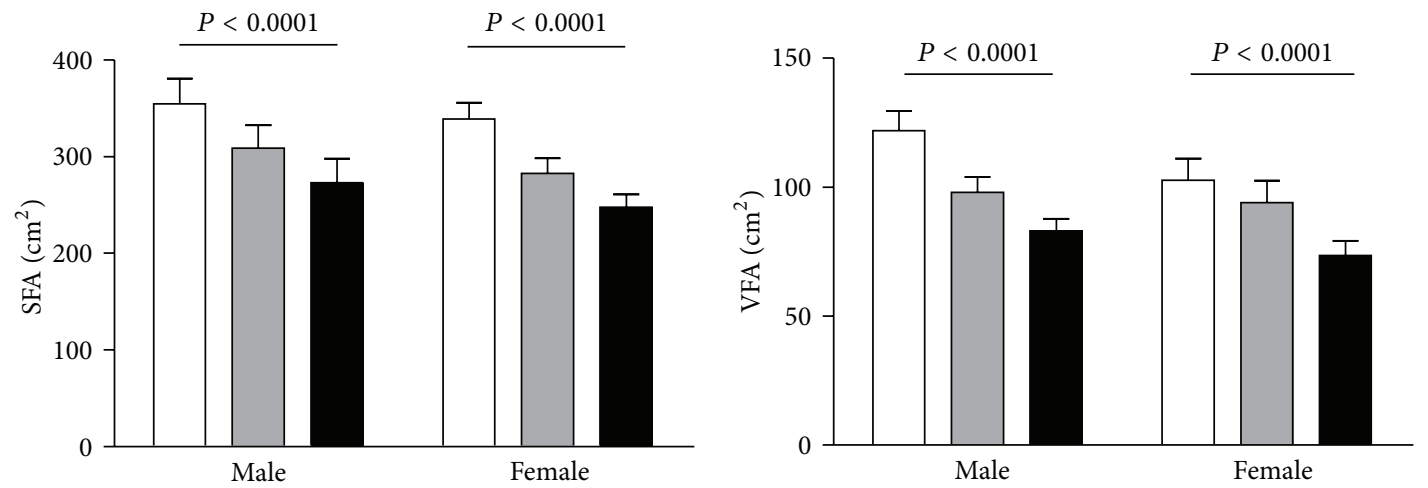

$\square$ Baseline
$\square \quad 4$ weeks
$\square 8$ weeks

$\square$ Baseline

$\square 4$ weeks

- 8 weeks

(a)
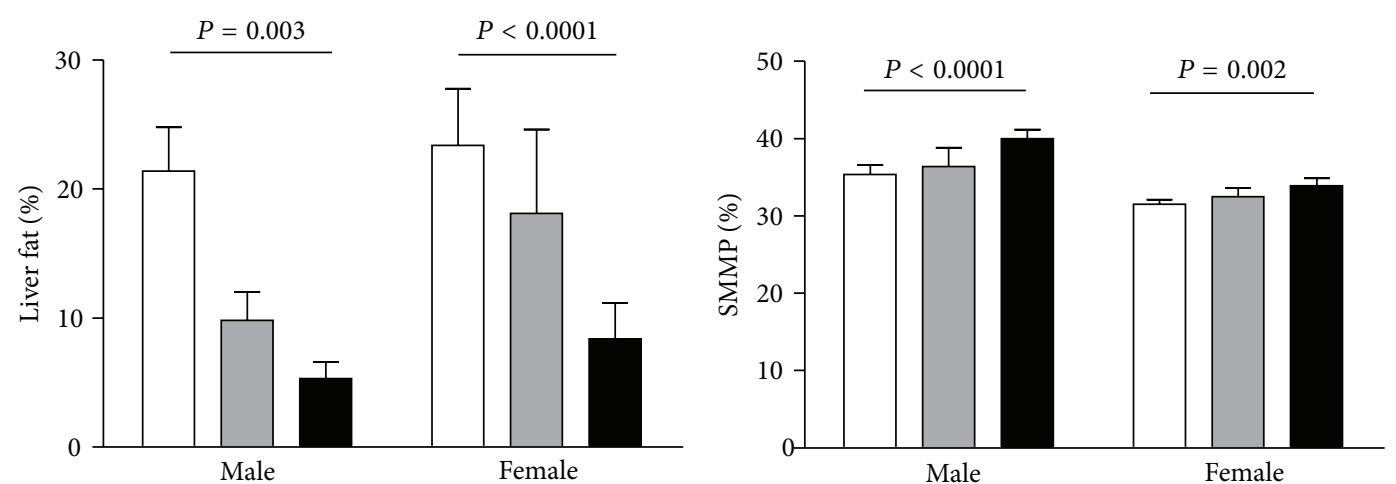

$\square$ Baseline

$\square$ Baseline

$\square 4$ weeks

$\square 4$ weeks

- 8 weeks

- 8 weeks

(c)

(d)

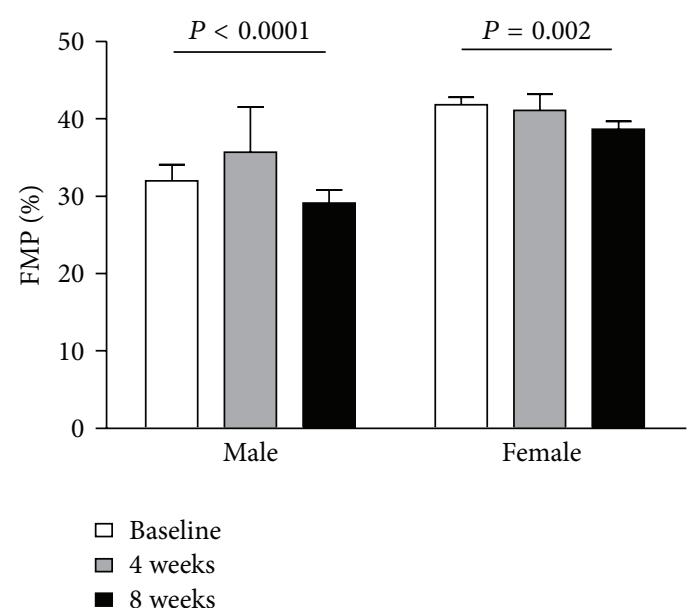

(e)

FIGURE 1: Improvement of body fat composition after an 8-week VLCD intervention. SFA: subcutaneous fat area; VFA: visceral fat area; SMMP: skeletal muscle mass percentage of whole body weight; VMP: fat mass percentage of whole body weight. 


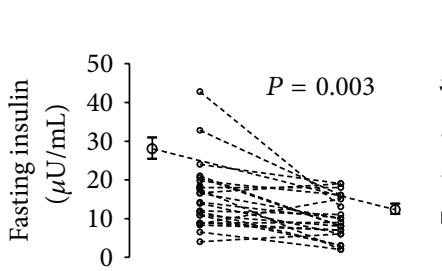

(a)

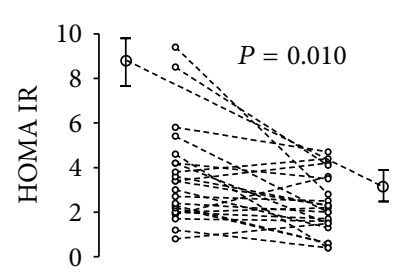

(e)

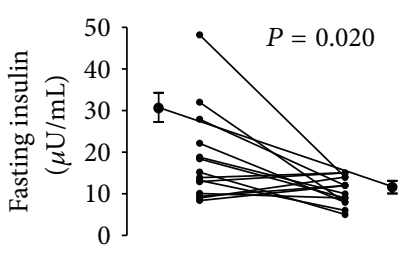

(b)

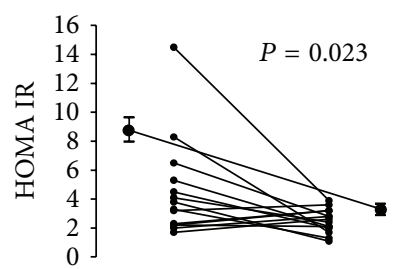

(f)

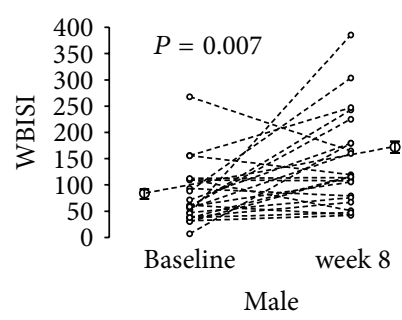

(i)

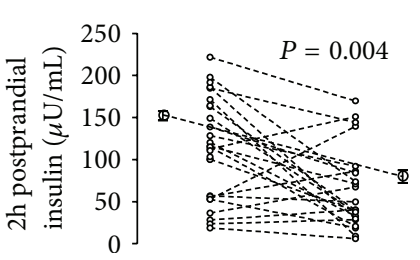

(c)

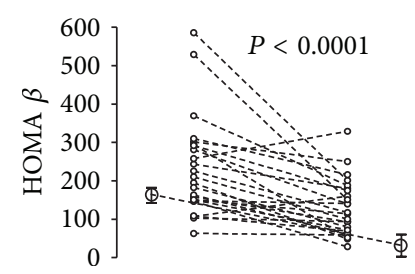

(g)

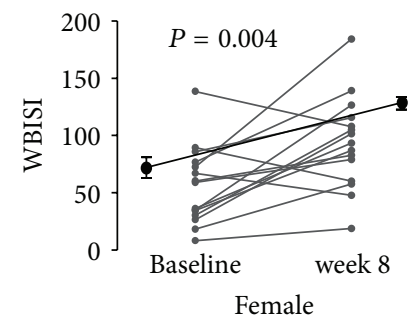

(j)

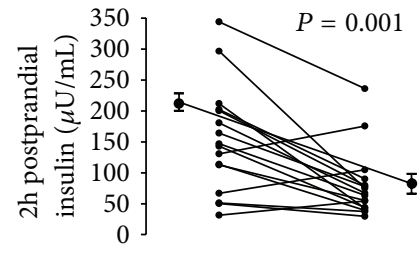

(d)

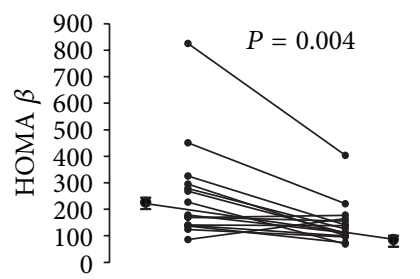

(h)

FIgURE 2: Changes of insulin resistance and sensitivity in male and female after 8 weeks of VLCD intervention. Solid lines represent the changes in male and dashed lines represent the changes in female.

were all improved in both male and female subjects (all $P$ values $<0.05)$.

\section{Discussion}

The major finding in the present study was that an 8-week VLCD intervention induced a significant weight loss with reduction of abdominal subcutaneous and visceral fat mass. This hypocarbohydrate dietary also had significant effects on liver fat content reduction and improvements of metabolic parameters and insulin responsiveness. These observations suggested that a VLCD diet intake resulted in a lower insulin/glucagon ratio, which enhanced adipose tissue lipolysis and oxidation with a subsequent promotion of liver ketone bodies production for energy needs [15].

In our study, we only observed a significant reduction of NEFA at week 8 . As a product of fat lipolysis and oxidation, BHA was in its peak level in week 4 and subsequently decreased in week 8 , with its concentration remaining significantly higher than that at baseline. Recently, a study reports that NEFA significantly increased in the first 7 days but decreased in week 8 in diabetic patients who participated in an 8-week VLCD intervention [16]. Therefore, we tentatively suppose that in our study, there might also exist a similar peak of NEFA caused by lipolysis at week 1 which we did not observe. Considering the changes of NEFA and BHA together, the two level curves might reflect the process of fat burning during the 8-week period.
It has long been presumed that weight loss after VLCD was associated with water loss [17] until Volek et al. applied dual-energy X-ray absorptiometry to examine the change of body composition in subjects who switched from their habitual diet ( $48 \%$ carbohydrate, $32 \%$ fat) to a VLCD $(8 \%$ carbohydrate, $61 \%$ fat) for 6 weeks [18]. They surprisingly found that fat mass significantly decreased and lean body mass significantly increased, despite no change in physical activity. Our results were similar to their findings that SMMP significantly increased and FMP significantly decreased at the end of the dietary intervention. It was suggested that water may account for some of the initial rapid weight loss, but fat loss subsequently accelerated and lean tissue was preserved over a longer period, which may be the main cause of weight loss [19].

After the 8-week dietary intervention, with the LFP significantly decreasing, half of the obese subjects with NAFLD had a reversal. Meanwhile, significant improvements of ALT, AST, and $\gamma$-GT were observed. Additionally, significant reduction of TC and TG and increment of HDL may have beneficial effects on the prevention of cardiovascular disease.

Several intervention studies and meta-analyses, varying in length from days to months have explored the impacts of VLCD diet on $\beta$ cell function and insulin sensitivity [2, $4,20,21]$. Most of the studies found that insulin sensitivity improved together with weight loss. In the present study, we found the similarities that HOMA IR, HOMA $\beta$, and WBISI were all significantly improved. There were several 
mechanisms involved in the amelioration of $\beta$ cell function and insulin sensitivity. One of the major hypothesis was that the reduction of triacylglycerol stores in pancreas and liver contributed to the normalization of $\beta$ cell function [16]. Furthermore, decreasing of triacylglycerol in skeletal muscle may be another cause of hepatic resistance alleviation [22]. However, the results from several studies demonstrated that insulin sensitivity did not alter after a short-term VLCD intervention for 5-7 days $[4,23]$. It might be a too short intervention period to detect the changes in insulin sensitivity [23].

In order to observe whether the effects of a VLCD were similar in different gender, we further analysed the body composition and insulin sensitivity in male and female, respectively. The results presented a similar beneficial effects in both genders.

The present study has some limitations. Firstly, we did not measure the metabolic parameters at the first week which may contribute to interpret the acute effect of VLCD. Secondly, for the lacking measurement of intramuscular triacylglycerol content, the relation between the change of it and improvement of insulin sensitivity was unclear. Finally, being a short-term designed study, the evidence of the longer effects of VLCD was unclear. So longer-term and further studies are needed to be designed to investigate the effects and to reveal the underlying mechanisms of VLCD in the future.

In conclusion, in both male and female, rapid weight reduction induced by an 8-week VLCD intervention effectively decreased total abdominal subcutaneous and visceral adipose tissue compartments, and liver fat content, increased skeletal muscle percentage of whole body weight, improved metabolic profile, and insulin resistance and sensitivity. All these beneficial effects might have been due to enhanced hepatic and whole-body lipolysis and oxidation.

\section{Authors' Contribution}

Y. Gu and $\mathrm{H}$. Yu contributed equally to this paper.

\section{Acknowledgments}

This study was supported by grants from national 973 program (2011CB504001), specific research fund from Ministry of Health (201002002), and national science and technology major project: significant creation of new drugs (2011zx09307-001-02). This study was funded in part by Nutriease Health Technology Co., Ltd.

\section{References}

[1] World Health Organization, "Diet, nutrition and the prevention of chronic diseases," Joint WHO/FAO Expert Consultation, WHO Technical Report Series no 916, WHO, Geneva, Switzerland, 2003.

[2] M. E. Frigolet, V. E. R. Barragan, and M. T. Gonzalez, "Lowcarbohydrate diets: a matter of love or hate," Annals of Nutrition and Metabolism, vol. 58, no. 4, pp. 320-334, 2011.

[3] P. F. Svendsen, F. K. Jensen, J. J. Holst, S. B. Haugaard, L. Nilas, and S. Madsbad, "The effect of a very low calorie diet on insulin sensitivity, beta cell function, insulin clearance, incretin hormone secretion, androgen levels and body composition in obese young women," Scandinavian Journal of Clinical and Laboratory Investigation, vol. 72, no. 5, pp. 410-419, 2012.

[4] I. Malandrucco, P. Pasqualetti, I. Giordani et al., "Very-lowcalorie diet: a quick therapeutic tool to improve beta cell function in morbidly obese patients with type 2 diabetes," The American Journal of Clinical Nutrition, vol. 95, no. 3, pp. 609613, 2012.

[5] A. P. M. Viljanen, R. Lautamäki, M. Järvisalo et al., "Effects of weight loss on visceral and abdominal subcutaneous adipose tissue blood-flow and insulin-mediated glucose uptake in healthy obese subjects," Annals of Medicine, vol. 41, no. 2, pp. 152-160, 2009.

[6] Y. Ahn, S. J. Park, H. K. Kwack, M. K. Kim, K. P. Ko, and S. S. Kim, "Rice-eating pattern and the risk of metabolic syndrome especially waist circumference in Korean genome and epidemiology study (KoGES)," BMC Public Health, vol. 13, no. 1, article 61, 2013.

[7] E. Völgyi, F. A. Tylavsky, A. Lyytikäinen, H. Suominen, M. Alén, and S. Cheng, "Assessing body composition with DXA and bioimpedance: effects of obesity, physical activity, and age," Obesity, vol. 16, no. 3, pp. 700-705, 2008.

[8] M. F. Xia, H. M. Yan, H. D. Lin et al., "Elevation of liver enzymes within the normal limits and metabolic syndrome," Clinical and Experimental Pharmacology and Physiology, vol. 38, no. 6, pp. 373-379, 2011.

[9] L. S. Szczepaniak, P. Nurenberg, D. Leonard et al., "Magnetic resonance spectroscopy to measure hepatic triglyceride content: prevalence of hepatic steatosis in the general population," The American Journal of Physiology, vol. 288, no. 2, pp. E462E468, 2005.

[10] B. Chowdhury, L. Sjostrom, M. Alpsten, J. Kostanty, H. Kvist, and R. Lofgren, "A multicompartment body composition technique based on computerized tomography," International Journal of Obesity, vol. 18, no. 4, pp. 219-234, 1994.

[11] M. R. Hoenig, G. Cowin, R. Buckley, C. McHenery, and A. Coulthard, "Liver fat percent is associated with metabolic risk factors and the metabolic syndrome in a high-risk vascular cohort," Nutrition and Metabolism, vol. 7, article 50, 2010.

[12] D. R. Matthews, J. P. Hosker, and A. S. Rudenski, "Homeostasis model assessment: insulin resistance and $\beta$-cell function from fasting plasma glucose and insulin concentrations in man," Diabetologia, vol. 28, no. 7, pp. 412-419, 1985.

[13] M. Fukushima, A. Taniguchi, M. Sakai et al., "Homeostasis model assessment as a clinical index of insulin resistance: comparison with the minimal model analysis," Diabetes Care, vol. 22, no. 11, pp. 1911-1912, 1999.

[14] M. Matsuda, Y. Liu, S. Mahankali et al., "Altered hypothalamic function in response to glucose ingestion in obese humans," Diabetes, vol. 48, no. 9, pp. 1801-1806, 1999.

[15] A. Adam-Perrot, P. Clifton, and F. Brouns, "Low-carbohydrate diets: nutritional and physiological aspects," Obesity Reviews, vol. 7, no. 1, pp. 49-58, 2006.

[16] E. L. Lim, K. G. Hollingsworth, B. S. Aribisala, M. J. Chen, J. C. Mathers, and R. Taylor, "Reversal of type 2 diabetes: normalisation of beta cell function in association with decreased pancreas and liver triacylglycerol," Diabetologia, vol. 54, no. 10, pp. 25062514, 2011.

[17] G. L. Blackburn, J. C. C. Phillips, and S. Morreale, "Physician's guide to popular low-carbohydrate weight-loss diets," Cleveland Clinic Journal of Medicine, vol. 68, no. 9, pp. 761-774, 2001. 
[18] J. S. Volek, M. J. Sharman, D. M. Love et al., "Body composition and hormonal responses to a carbohydrate-restricted diet," Metabolism, vol. 51, no. 7, pp. 864-870, 2002.

[19] J. S. Volek and E. C. Westman, "Very-low-carbohydrate weightloss diets revisited," Cleveland Clinic Journal of Medicine, vol. 69, no. 11, pp. 849-858, 2002.

[20] G. D. Foster, H. R. Wyatt, J. O. Hill et al., "A randomized trial of a low-carbohydrate diet for obesity," The New England Journal of Medicine, vol. 348, no. 21, pp. 2082-2090, 2003.

[21] I. Shai, D. Schwarzfuchs, Y. Henkin et al., "Weight loss with a low-carbohydrate, Mediterranean, or low-fat diet," The New England Journal of Medicine, vol. 359, no. 3, pp. 229-241, 2008.

[22] L. G. Grunnet, E. Laurila, O. Hansson et al., "The triglyceride content in skeletal muscle is associated with hepatic but not peripheral insulin resistance in elderly twins," Journal of Clinical Endocrinology and Metabolism, vol. 97, no. 12, pp. 4571-4577, 2012.

[23] C. C. Wang, R. L. Adochio, J. W. Leitner, I. M. Abeyta, B. Draznin, and M. A. Cornier, "Acute effects of different diet compositions on skeletal muscle insulin signalling in obese individuals during caloric restriction," Metabolism, 2012. 


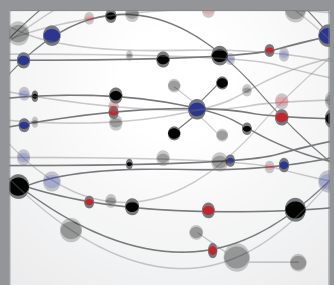

The Scientific World Journal
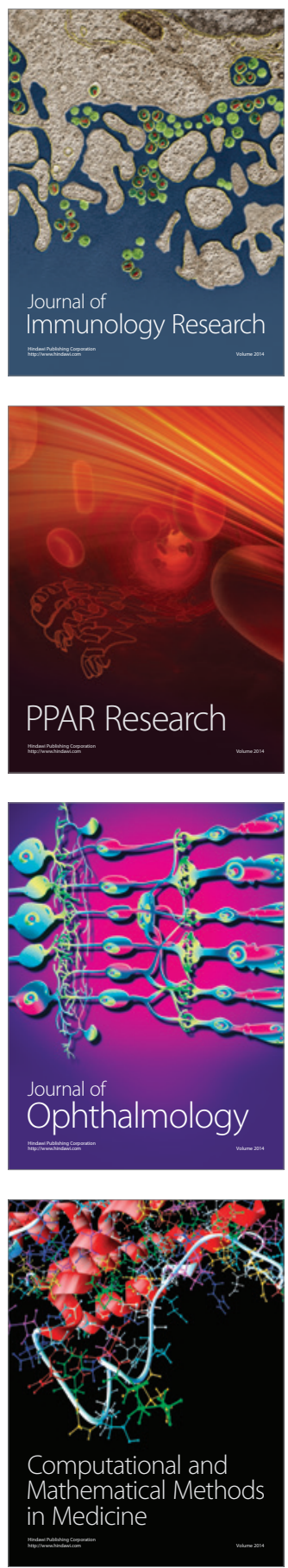

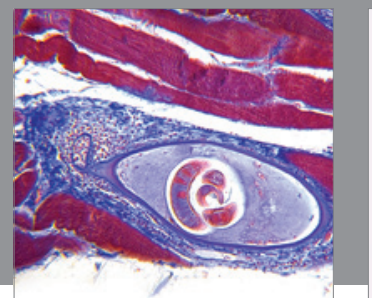

Gastroenterology

Research and Practice
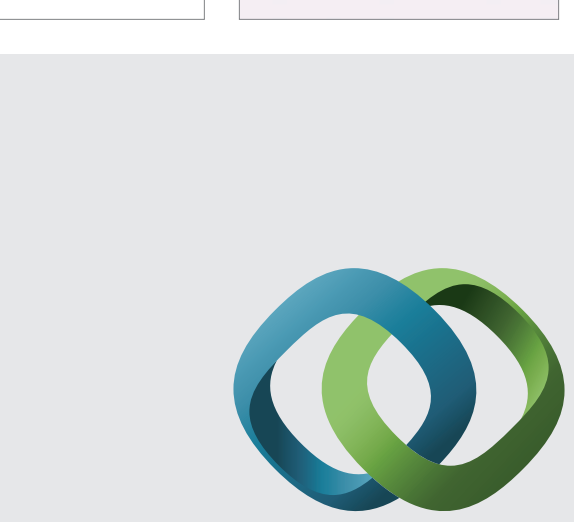

\section{Hindawi}

Submit your manuscripts at

http://www.hindawi.com
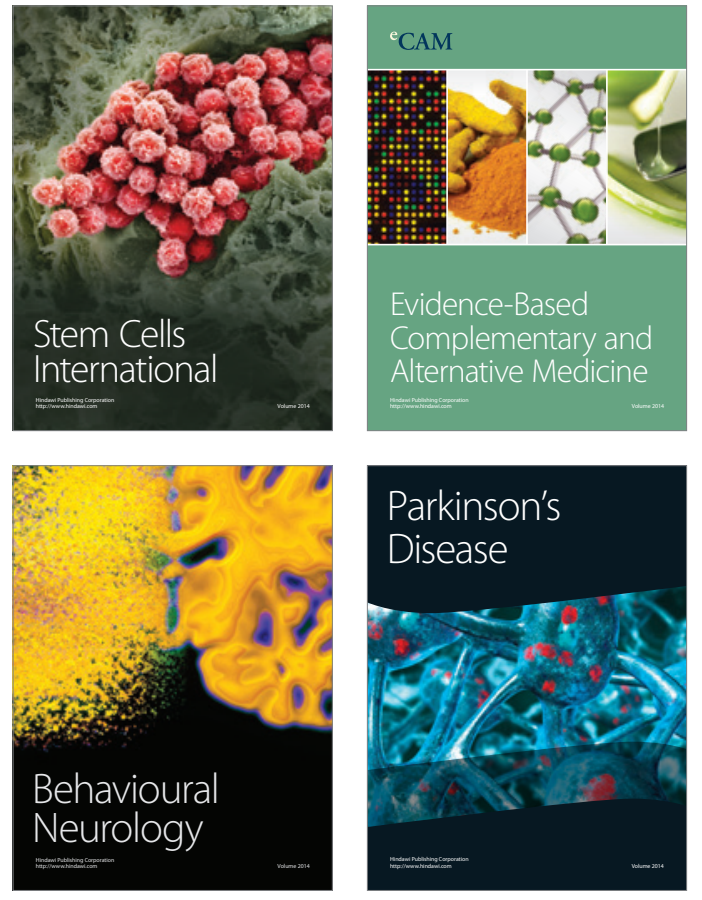
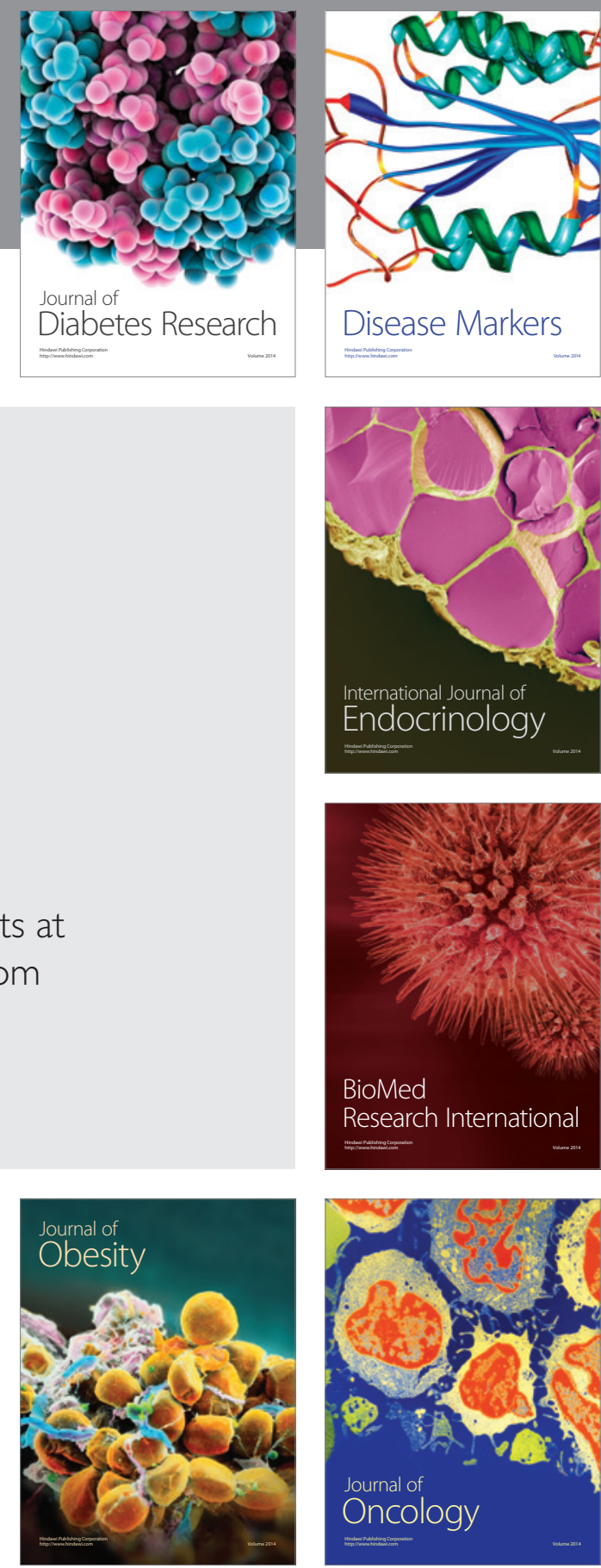

Disease Markers
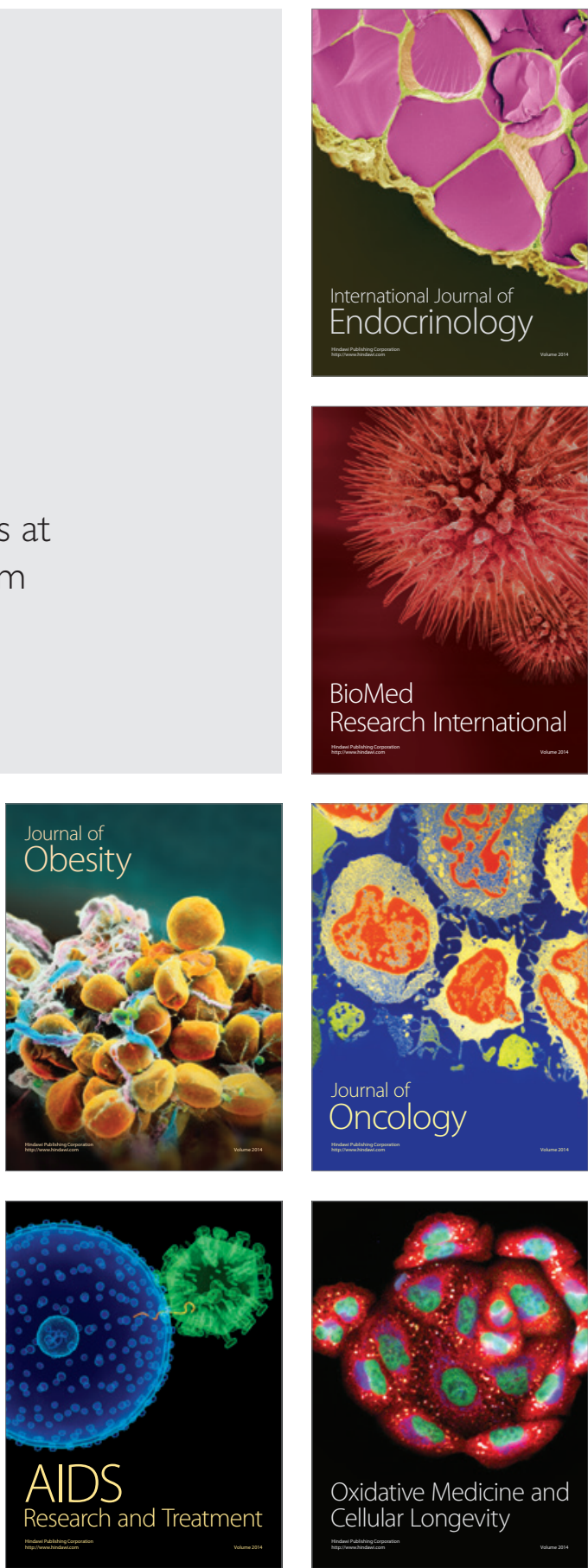